\title{
Acute Interstitial Lung Disease Induced by Rechallenge with Ceritinib
}

\author{
Takamasa Hotta, Tamio Okimoto, Megumi Hamaguchi, Yukari Tsubata and Takeshi Isobe
}

\begin{abstract}
:
A 40-year-old Japanese man with advanced pulmonary adenocarcinoma harboring anaplastic lymphoma kinase (ALK)-rearranged was administered the selective ALK inhibitor ceritinib as a third-line treatment and continued treatment for nine months. After fourth-line treatment, we performed rechallenge with ceritinib as a fifth-line treatment. On day 54 after rechallenge, the patient developed acutely deteriorating dyspnea. Chest computed tomography showed extensive ground-glass opacities. We diagnosed him with ceritinib-induced interstitial lung disease (ILD) and initiated methylprednisolone pulse therapy. To our knowledge, this is the first report of ceritinib-induced ILD in a Japanese patient. Since it may newly emerge with rechallenge therapy, close attention is necessary.
\end{abstract}

Key words: ceritinib, interstitial lung disease, anaplastic lymphoma kinase, adverse event

(Intern Med 59: 253-256, 2020)

(DOI: 10.2169/internalmedicine.2597-18)

\section{Introduction}

Anaplastic lymphoma kinase (ALK)-tyrosine kinase inhibitors (TKIs) have superior efficacy to cytotoxic agents $(1,2)$, ALK-TKIs are commonly used for ALK fusion protein-positive non-small cell lung cancer (NSCLC). Crizotinib was the only drug that had been approved for treatment of ALK-rearranged NSCLC as of 2013. The second-generation ALK-TKIs alectinib and ceritinib are novel, highly selective oral ALK-TKIs that are extremely active in patients with advanced ALK-rearranged NSCLC, including those who experienced disease progression during crizotinib treatment $(3,4)$.

Interstitial lung disease (ILD) is a rare complication during ALK-TKI treatment. Regarding crizotinib-induced ILD, across 1,397 cases investigated in clinical trials, 34 patients (2.4\%) had any-grade ILD, $13(0.9 \%)$ had grade 3 or 4 ILD, and $7(0.5 \%)$ died (5). Regarding alectinib-induced ILD, 1 patient developed grade 1 ILD in a Phase 1-2 study ( $\mathrm{n}=$ 70) (6). In clinical studies of ceritinib, ILD/pneumonitis was reported in $2.4 \%$ of 925 patients (7). There have been reports of successful rechallenge therapy with crizotinib or alectinib after the development of ALK-TKI-induced
$\operatorname{ILD}(8,9)$. However, there are no previous reports of $\operatorname{ILD}$ associated with ceritinib.

To our knowledge, this is the first report to include detailed clinicopathological information on ceritinib-induced ILD.

\section{Case Report}

A 40-year-old Japanese man presented to our hospital with pulmonary adenocarcinoma. RNA sequencing revealed the presence of the EML4-ALK fusion protein. When he was diagnosed, he rejected all our recommendations for chemotherapy and received only whole-brain radiation therapy. Six months after the initial diagnosis, he had cardiac tamponade. He received pericardiocentesis and agreed to start chemotherapy. Crizotinib and alectinib were administered as first- and second-line treatments, respectively. We selected ceritinib $(750 \mathrm{mg}$, once daily) as a third-line treatment. Although treatment was discontinued once due to leukocytoclastic vasculitis, we continued ceritinib with strict follow-up observation (10). At nine months after the initiation of ceritinib, progressive disease was detected. Carboplatin, pemetrexed and bevacizumab therapy were administered as a fourth-line treatment. After chemotherapy, the patient devel- 


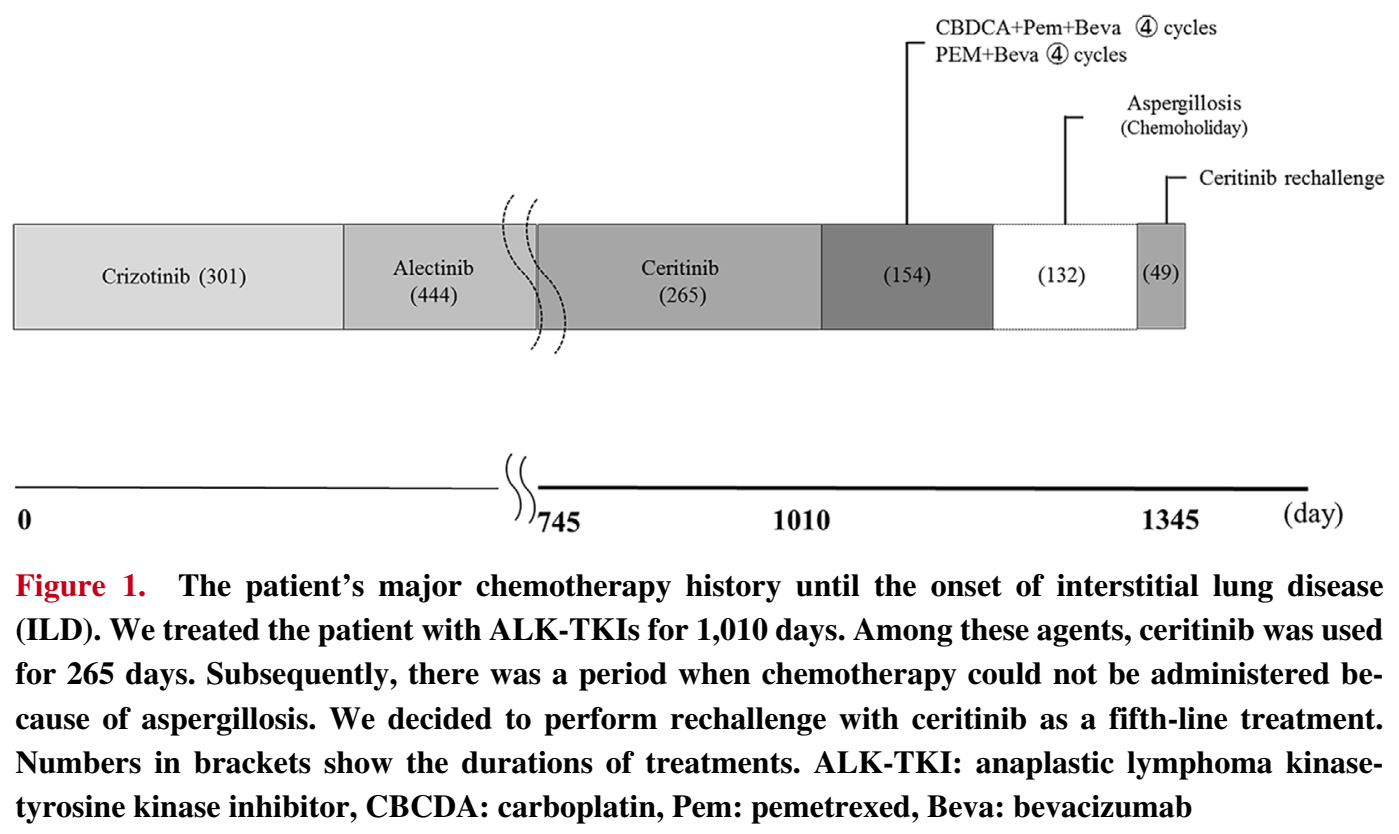

oped aspergillosis. Chemotherapy was discontinued, and antifungal medication (Voriconazole) was administered.

As the disease progressed (brain metastasis), the patient expressed his wish to retry ceritinib treatment. Because of aspergillosis, we were hesitant to administer cytotoxic chemotherapy. During the previous treatments, crizotinib had induced nausea, alectinib had induced liver dysfunction, and ceritinib had induced leukocytoclastic vasculitis. However, when the patient had been administered ceritinib, his brain metastasis and leukocytoclastic vasculitis had been able to be controlled. We therefore performed a rechallenge with ceritinib as a fifth-line treatment (Fig. 1).

Voriconazole was discontinued on day 28 after the rechallenge due to liver dysfunction. On day 49 after the rechallenge, we discontinued ceritinib due to the development of liver dysfunction. The patient's serum levels were as follows: T-Bil (0.5 mg/dL), AST (336 IU/L), ALT (456 IU/L), ALP (346 IU/L), and $\gamma$-GTP (170 IU/L). On day 54 after rechallenge, the patient developed acutely deteriorating dyspnea without demonstrable infection. Chest computed tomography (CT) showed extensive ground-glass opacities throughout the left lung (Fig. 2A). A laboratory analysis revealed the following findings: LDH, $394 \mathrm{IU} / \mathrm{L}$; KL-6, 312 $\mathrm{U} / \mathrm{mL}$; and SP-D, $244.0 \mathrm{ng} / \mathrm{mL}$. Methylprednisolone pulse therapy ( $1 \mathrm{~g}$ once per day for 3 days) was thus initiated. By day 4 after admission, the patient's respiratory status had begun to improve. After day 4 of prednisolone $(60 \mathrm{mg}$ per day) treatment, methylprednisolone pulse therapy was performed a second time. Subsequently, his imaging findings and serum levels of KL-6, SP-D, and LDH gradually improved (Fig. 2B). The hepatic dysfunction also recovered to normal values on the 15th day after discontinuing ceritinib (64 days after rechallenge) (Fig. 3). By day 15 after admission, oxygen became unnecessary. The steroid dose was gradually tapered, while ceritinib was kept withdrawn. Flexible bronchoscopy revealed no evidence of microorganisms,

fungi, Pneumocystis jirovecii, or malignancy. The echocardiography findings showed a normal cardiac function. We diagnosed him with ceritinib-induced interstitial lung disease (ILD). By day 17 after admission, he was discharged from our hospital. He died due to exacerbation of brain metastasis, but no recurrence of ILD was detected.

\section{Discussion}

This is, to our knowledge, the first report of ceritinibinduced ILD due to rechallenge therapy. The radiological patterns of drug-induced ILD can be divided into five main types: diffuse alveolar damage (DAD), hypersensitivity reaction, nonspecific interstitial pneumonia, organizing pneumonia (OP), and eosinophilic pneumonia (11). In the present case, at the time of the rechallenge, the diffuse ground-grass attenuation of dorsal side advantage DAD pattern was observed. DAD is subdivided into exudative, organizing, and fibrotic phases. This case had an early-stage DAD pattern. Early treatment intervention was successful.

Certain factors have been reported to be associated with an increased risk or the occurrence of drug-induced lung injury. Nonspecific risk factors include existing pulmonary lesions (interstitial pneumonia in particular), age $>60$ years old, a poor respiratory function, a history of pulmonary surgery, the administration of oxygen, irradiation to the lungs, and the presence of renal impairment $(12,13)$. A decreased kidney function is also a risk factor in the sense that it increases the blood concentration of the drug.

One possible reason for the development of severe interstitial pneumonia was the increasing blood concentration of ceritinib. Renal excretion is as low as $1.3 \%$, and ceritinib is thought to be eliminated from the liver mainly by metabolism and bile excretion. In patients with liver dysfunction the metabolism of ceritinib may be decreased, which would increase exposure (7). Regarding drug interactions, CYP3A 


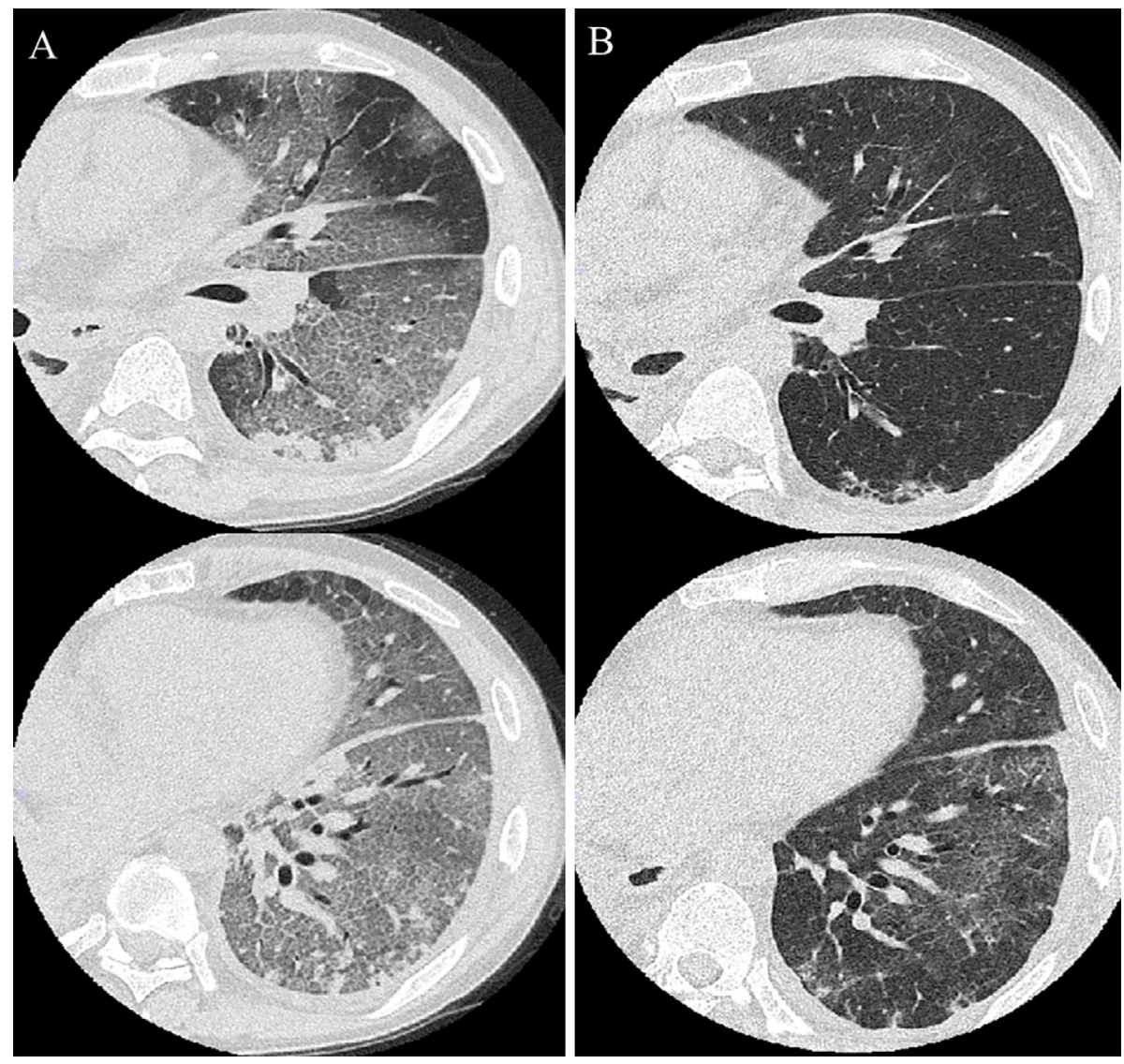

Figure 2. The chest computed tomography findings. Chest computed tomography on day 54 after the start of rechallenge therapy revealed interstitial lung disease (A). On day 15 after admission, the ground-glass opacities gradually improved (B).

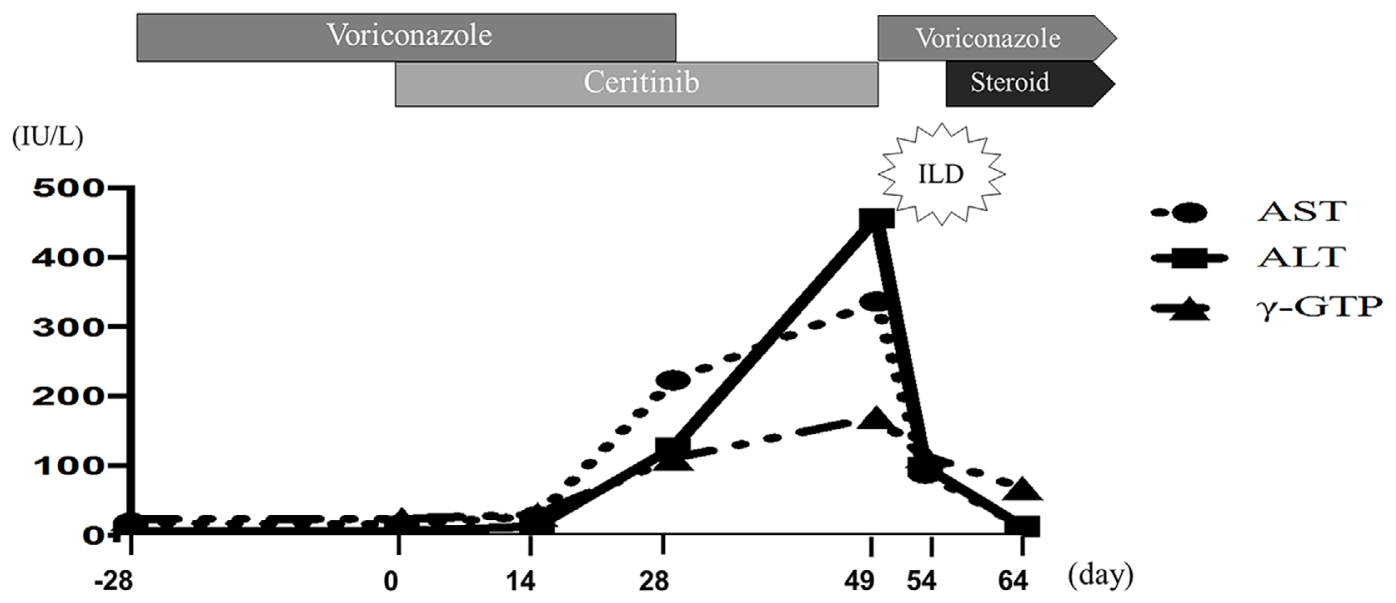

Figure 3. Transition of liver dysfunction and drug use history. The day of rechallenge of ceritinib is considered to be day 0 . Although the liver function was normal when voriconazole was used alone, liver dysfunction was observed on day 28 when voriconazole was used in combination with ceritinib. We discontinued voriconazole administration, but his liver dysfunction worsened, and ceritinib was discontinued as well on day 49. Withdrawal of ceritinib improved the liver dysfunction, but interstitial lung disease (ILD) developed. The liver function normalized at day 64.

is a major metabolic enzyme involved in the metabolism of ceritinib, so its maximum concentration and area under the blood concentration-time curve are increased by CYP3A inhibitors (e.g., ketoconazole). Voriconazole, which was resumed with the withdrawal of ceritinib, has a strong inhibi- tory action on CYP3A. The ceritinib concentration may have been sharply increased by liver dysfunction and the combination of voriconazole.

Epidermal growth factor receptor (EGFR)-TKIs (gefitinib and erlotinib) in advanced NSCLC significantly increase the 
risk of developing both all-grade and fatal ILD events (14). The use of ALK-TKIs also significantly increases the risk of developing high-grade ILD (15). At present, the specific mechanism underlying TKI-induced ILD is unclear. A previous study found that the EGFR signaling pathway was associated with the repair of airway epithelial cells. The inhibition of such repair signaling leads to prolonged inflammation, potentiating acute lung injury (16). However, despite these findings concerning EGFR-TKI-induced ILD, the mechanism underlying ALK-TKI-induced ILD remains unclear.

As a limitation associated with this case, we were unable to perform a lung biopsy because the right lung was not functioning sufficiently due to cancer and aspergillosis. Regarding the differential diagnosis of the chest CT findings, the bronchoalveolar lavage fluid was negative for infection, including fungal and $P$. jirovecii. The possibility of virus infection was deemed to be low because there were no symptoms of lower respiratory infection. In addition, the possibility of cardiogenic pulmonary edema was excluded based on the echocardiography findings showing a normal left ventricular systolic and diastolic function. For the treatment, in addition to steroid therapy, we used tazobactam/piperacillin only for the first three days, and no diuretics or antiviral drugs were required. Voriconazole was continued, and ceritinib was the only medication discontinued during ILD treatment.

In conclusion, just because the initial treatment is successful does not mean that rechallenge will also be successful. Careful follow-up observation is necessary when using drugs that are associated with concerns of drug interactions.

\section{Author's disclosure of potential Conflicts of Interest (COI).}

Takeshi Isobe: Honoraria, Boehringer Ingelheim, AstraZeneca and Pfizer.

\section{References}

1. Solomon BJ, Mok T, Kim DW, et al. First-line crizotinib versus chemotherapy in ALK-positive lung cancer. N Engl J Med 371: 2167-2177, 2014.

2. Shaw AT, Kim DW, Nakagawa K, et al. Crizotinib versus chemotherapy in advanced ALK-positive lung cancer. N Engl J Med 368: 2385-2394, 2013.
3. Shaw AT, Gandhi L, Gadgeel S, et al. Alectinib in ALK-positive, crizotinib-resistant, non-small-cell lung cancer: a single-group, multicentre, phase 2 trial. Lancet Oncol 17: 234-242, 2016.

4. Shaw AT, Kim DW, Mehra R, et al. Ceritinib in ALK-rearranged non-small-cell lung cancer. N Engl J Med 370: 1189-1197, 2014.

5. Créquit $\mathrm{P}$, Wislez M, Fleury Feith J, et al. Crizotinib associated with ground-glass opacity predominant pattern interstitial lung disease: a retrospective observational cohort study with a systematic literature review. J Thorac Oncol 10: 1148-1155, 2015.

6. Seto T, Kiura K, Nishio M, et al. CH5424802 (RO5424802) for patients with ALK-rearranged advanced non-small-cell lung cancer (AF-001JP study): a single-arm, open-label, phase 1-2 study. Lancet Oncol 14: 590-598, 2013.

7. Zykadia ${ }^{\circledR}$ (ceritinib) adverse reactions \& safty profile [Internet]. [cited 2018 Dec 28]. Available from: https://www.hcp.novartis.co m/products/zykadia/alk-nsclc/safety-profile/

8. Yanagisawa S, Inoue A, Koarai A, Ono M, Tamai T, Ichinose M. Successful crizotinib retreatment after crizotinib-induced interstitial lung disease. J Thorac Oncol 8: 73-74, 2013.

9. Chino H, Sekine A, Kitamura H, Kato T, Ogura T. Successful treatment with alectinib after crizotinib-induced interstitial lung disease. Lung Cancer 90: 610-613, 2015.

10. Okimoto T, Tsubata $Y$, Hotta $T$, et al. Successful rechallenge with ceritinib after leukocytoclastic vasculitis during ceritinib treatment for non-small cell lung cancer harboring the EML4-ALK fusion protein. Oncotarget 9: 20213-20218, 2018.

11. Sakai F, Johkoh T, Kusumoto M, Arakawa H, Takahashi M. Druginduced interstitial lung disease in molecular targeted therapies: high-resolution CT findings. Int J Clin Oncol 17: 542-550, 2012.

12. Kubo A, Azuma A, Kanazawa M, et al. Consensus statement for the diagnosis and treatment of drug-induced lung injuries. Respir Investig 51: 260-277, 2013.

13. Camus $P$, Kudoh $S$, Ebina M. Interstitial lung disease associated with drug therapy. Br J Cancer 91: 18-23, 2004.

14. Shi L, Tang J, Tong L, Liu Z. Risk of interstitial lung disease with gefitinib and erlotinib in advanced non-small cell lung cancer: a systematic review and meta-analysis of clinical trials. Lung Cancer 83: 231-239, 2014.

15. Lin L, Zhao J, Kong N, et al. Meta-analysis of the incidence and risks of interstitial lung disease and QTc prolongation in nonsmall-cell lung cancer patients treated with ALK inhibitors. Oncotarget 8: 57379-57385, 2017.

16. Harada C, Kawaguchi T, Ogata-Suetsugu S, et al. EGFR tyrosine kinase inhibition worsens acute lung injury in mice with repairing airway epithelium. Am J Respir Crit Care Med 183: 743-745, 2011.

The Internal Medicine is an Open Access journal distributed under the Creative Commons Attribution-NonCommercial-NoDerivatives 4.0 International License. To view the details of this license, please visit (https://creativecommons.org/licenses/ by-nc-nd/4.0/).

(C) 2020 The Japanese Society of Internal Medicine Intern Med 59: 253-256, 2020 